\title{
A technique for obtaining 3D coordinates of human body parts from RGB-D stream
}

\author{
Alexander V. Fisunov*, Victoria B. Gnezdilova, and Vladimir I. Marchuk \\ Don State Technical University, 346500 Shakhty, Russia
}

\begin{abstract}
This paper proposes the method for real time determining three-dimensional coordinates of human body parts from RGB-D stream. Proposed method represent a combined solution in which deep learning and depth map analysis are used.
\end{abstract}

\section{The statement of the problem}

Understanding human pose and body parts 3D coordinates is an important task in computer vision. There are many related works in this field, but progress is still required for accurate real-time pose estimation. The task of human pose estimation is relevant to motion capture without markers, augmented reality, analysis of body parts motion for medical purposes, analysis of human behavior, remote control of multimedia systems, etc.

Existing solutions of human pose estimation from RGB or RGB-D stream have a number of issues: inability to work in real-time and high requirements to hardware, low probability of body parts detection, negative influence of different environment conditions in the process of videorecording, etc. Currently, there is no single solution to all these problems.

\section{The proposed method}

In this paper, we propose a combined method that combines the use of deep learning algorithms based on the Google Object Detection API and mathematical analysis of RGB-D stream. A trained neural network searches for body parts in an RGB image. Due to the chosen model of the neural network and the collected database of images, there is a possibility that some of the parts cannot be detected (for example, the elbow or the neck), including a mathematical module and analyzing the depth map finds this part of the body.

Figure 1 shows the algorithm of the proposed method, on the right shows the preparatory stage of neural network training, on the left - the main cycle of RGB-D stream processing and the result is the three-dimensional coordinates of human body parts in space.

\footnotetext{
*Corresponding author: fisunov.a.v@gmail.com
} 

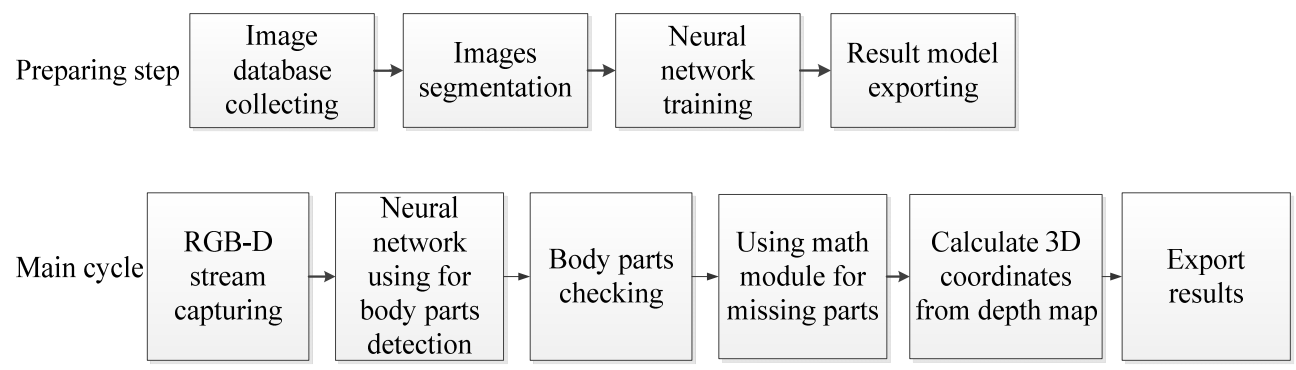

Fig. 1. The block diagram of the algorithm of the proposed method

For training the neural network, the authors collected a database of 4000 images, in which there are from 1 to 3 people. The following parts of the body were marked in these images: head, neck, shoulders, elbows, hands and torso; for each part, 2500 fragments were obtained.

The marking process does not specify which side, that is, the left and right hand, marked as "hand", to determine which of the analysis of the human skeleton on the found points. Figure 2 shows examples of marked images from the database.

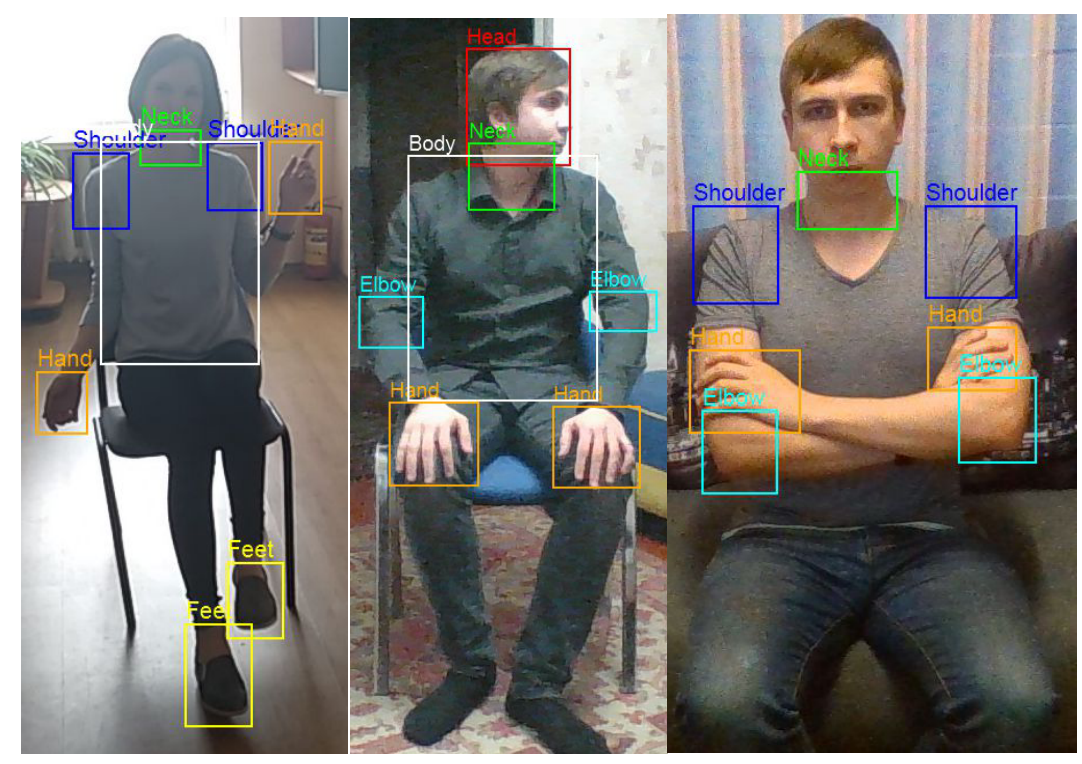

Fig. 2. Examples of marked images used for training a neural network.

The model ssd_mobilenet_v1_coco was chosen as a training model due to the high speed of its operation, which is necessary for tracking a person's posture in real time. The training was conducted in the Amazon cloud and consisted of 225,000 steps.

Let's consider now the algorithm of the main cycle of the analysis of human posture. In the first step, using a neural network, the main parts of the body are detected on the RGB image and those that have not been found are marked. For missed objects, a different mathematical detection module is included as described below.

If the head isn't detected, the Haar cascade algorithm [1] searches for the face, since in most cases the head (or face) is the starting point for detecting the rest parts of the body.

To find the neck, it is enough to check the area below the head in the depth map, calculating the value of the depth gradient (equation 1) [2] in this area. At that point where the gradient exceeds the threshold, the center of the neck is marked. 


$$
\nabla f=\left[\frac{g_{x}}{g_{y}}\right]=\left[\begin{array}{l}
\frac{\partial f}{\partial x} \\
\frac{\partial f}{\partial y}
\end{array}\right]
$$

Knowing the proportions of the human body, helps in determining the height at which the shoulders are located, and moving to the sides along the $\mathrm{x}$ coordinate from the neck also we find the points which exceed a given gradient, this will be the coordinates of the center of the shoulders.

Knowing the coordinates of the shoulders in the depth map, with the help of the fire extinguishing line method [2, 3, 4], the structure of human hands (Formula) is constructed. The correctness of the construction which is checked by calculation of the ratio of the lengths of the shoulder-elbow and elbow-wrist.

The transition from RGB to depth space and vice versa was carried out on the basis of information about the internal and external parameters of the used camera which is the Intel RealSense SR300 camera. Such transitions are necessary due to the fact that the body parts can be found both on the RGB image using a neural network, and methods of the mathematical analysis. In addition, even if you find a point in the RGB image, you need to translate it to a depth map to obtain the resulting 3D coordinates.

In case of the loss of tracking by the detector, the coordinates obtained in the past are used for the next few frames.

\section{The obtained results}

Figure 3 shows examples of RGB-D stream processing by the proposed method. Large rectangles show the parts of the body found by the neural network, small rectangles show the centers of the body parts calculated by the depth map. For each area, the position in the RGB image, the distance to it, and the name of the body part to which this area refers are indicated.

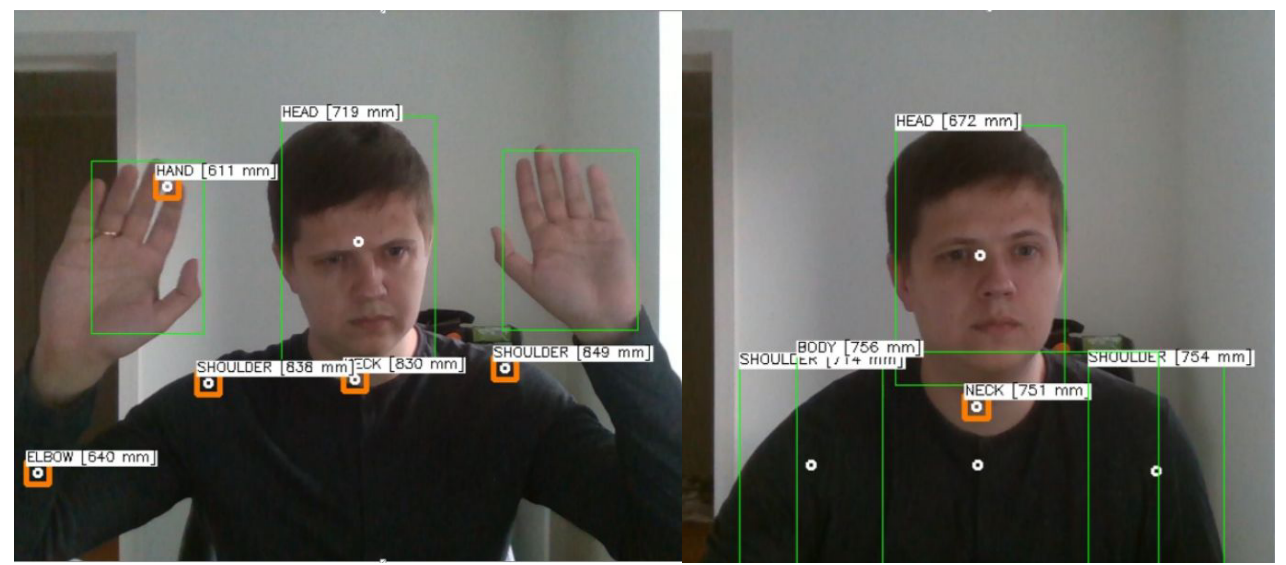

Fig. 3 Examples of detection of body parts in RGB-D stream.

For an RGB image with a resolution of $1920 \times 1080$ and a depth map of $640 \times 480$, it takes $35-45 \mathrm{~ms}$ to process one frame using an Intel Core i5 $45703.2 \mathrm{GHz}$ processor. Disabling the module that uses the neural network, and only using the mathematical analysis of RGB-D stream, the processing speed increases to $20-25 \mathrm{~ms}$ per frame.

To study the accuracy of the work, a comparison was made with solution for determining the human posture of OpenPose, the results of a comparison are present in table 1. 
Table 1. The results of methods accuracy comparison

\begin{tabular}{|l|l|l|l|l|l|l|}
\hline Body part & Hand & Head & Body & Shoulders & Elbows & Neck \\
\hline $\begin{array}{l}\text { The probability of detection } \\
\text { using the proposed method, } \%\end{array}$ & 88 & 98 & 86 & 84 & 65 & 96 \\
\hline $\begin{array}{l}\text { The probability of detection } \\
\text { using OpenPose, } \%\end{array}$ & 100 & 100 & 99 & 99 & 99 & 99 \\
\hline
\end{tabular}

The reduced probability of detecting elbows in comparison with the rest of the body is due to the lack of special signs of elbows, they can be similar to anything. The high probability of detection of OpenPose is associated with a very large database of images for training, several stages of verification and high requirements for the computing power of the device.

\section{Conclusion}

In this paper, a method is proposed that makes it possible to detect human body parts on an RGB-D flow in real time and obtain their spatial 3D coordinates.

In comparison with the OpenPose method, the proposed method loses not more than $20 \%$ on average, however it allows working in real time, with a frequency of 30 frames per second for Full HD video stream and depth maps with a resolution of $640 \times 480$. OpenPose, when using the same hardware, operates not in real-time.

\section{References}

1. P. Viola, M. Jones, IEEE Conf Comput Vis Pattern Recognit, 1, I-511 (2001) doi: 10.1109/CVPR.2001.990517

2. R.C. Gonzalez, R.E. Woods, Digital image processing second edition (Publishing House of Electronics Industry, Beijing, 2002)

3. U. Montanari, JACM, 15 (4), 600 (1968)

4. U. Montanari, JACM, 16 (4), 534 (1969) 